\title{
Epstein-Barr Virus (EBV) Prevalence and the Risk of Reactivation in Patients with Inflammatory Arthritis Using Anti-TNF Agents and in those who are Biologic Naive
}

\author{
Emily McKeown ${ }^{1}$, Janet E. Pope ${ }^{*}, 2$ and Suzanne Leaf ${ }^{2}$ \\ ${ }^{I}$ Department of Medicine, London Health Sciences Centre, London, Ontario, Canada \\ ${ }^{2}$ Division of Rheumatology, St. Joseph's Health Centre, London, Ontario, Canada
}

\begin{abstract}
Objective: Anti-TNF agents (etanercept, infliximab and adalimumab) are widely used in inflammatory conditions, such as rheumatoid arthritis; however, they are not without side effects, potentially including lymphoma. We compared Epstein-Barr virus (EBV) levels in patients with inflammatory arthritis taking biologic agents and controls matched for disease, age, gender and disease duration who were biologic naïve. Secondly, we determined the risk of reactivation of $\mathrm{EBV}$ in patients taking biologics.

Methods: One hundred and twenty-two patients were recruited and blood samples were collected. Immunoglobulin G (IgG) antibody to EBV was analysed using enzyme-linked immunosorbent assay. EBV DNA was analysed using polymerase chain reaction (PCR) on all positive IgG samples. Quantitative measures of viral DNA were made and expressed as copies/reaction volume. Reactivation was defined as the presence of viral DNA in the plasma and PCR activity was evaluated between 6 and 18 months after anti-TNF therapy.

Results: IgG for EBV was detected in $98 \%$ of controls and $90 \%$ of cases. Viral reactivation related to EBV was not observed in this study. There was one patient who tested positive for EBV using PCR, but upon confirmatory testing, this sample was actually negative. No samples were positive on PCR at the follow-up time points.

Conclusion: There was a high rate of EBV IgG in the cases and controls in this study. Given the small sample size and timeframe for this study, treatment with anti-TNF agents does not seem to lead to EBV reactivation, and thus, this is likely not a mechanism for the development of lymphoma in patients taking biologics.
\end{abstract}

Keywords: Rheumatoid arthritis, anti-TNF therapy, reactivation, Epstein-Barr Virus, lymphoma.

\section{INTRODUCTION}

Rheumatoid arthritis (RA) is characterized by a systemic inflammatory disorder that causes synovitis leading to joint destruction. Research into the pathogenesis has shown that an abnormal immune response in a genetically predisposed patient is the hallmark of the disease. Specifically, tumor necrosis factor (TNF)-alpha $(\alpha)$ has been shown to be involved in the inflammatory cascade due to the production of many cytokines and studies have demonstrated clinical benefit with TNF- $\alpha$ blockade [1-4]. In patients who have not responded to traditional disease-modifying agents (DMARDs), these biologic agents have become an important option in the rheumatologist's repertoire to treat RA $[3,5,6]$. There are three anti-TNF drugs on the market in Canada, etanercept, infliximab and adalimumab, and all target the proinflammatory cytokine, tumour necrosis factor. Infliximab is a mouse human chimeric antibody to TNF-alpha and adalimumab is a fully human chimeric antibody. Etanercept is a fusion protein that binds TNF and has also been shown to neutralize lymphotoxin alpha which is associated with

*Address correspondence to this author at the Division of Rheumatology, St. Joseph's Health Centre, 268 Grosvenor St., London, ON N6A 4V2, Canada; Tel: 519-646-6332; Fax: 519-646-6334;

E-mail: janet.pope@sjhc.london.on.ca tumour growth control [5]. These agents have proven efficacy in the treatment of RA, psoriatic arthritis and ankylosing spondylitis [5]. However, there are important clinical concerns about their side effects including risk of infection, lymphoma, reactivation of tuberculosis, and demyelinating syndromes [6-10]. Evidence from clinical trials and postmarketing surveys suggest that there may be an increased risk of lymphoma, however the mechanism for oncogenicity is not entirely known [11, 12]. Wolfe and Michaud published data on 18,574 patients with RA in which there were 29 lymphomas; the standardized incidence ratio (SIR) for lymphoma was 1.9 (95\% CI 1.3-2.7). In patients receiving biologics, the SIR was 2.9 (95\% CI 1.7-4.9) [7]. However, the confidence intervals overlap and it is difficult to know if disease severity is the added risk that further heightens the observed number of lymphomas in anti-TNF treatment above overall RA background risk.

One of the proposed mechanisms for the observed increased risk of malignancy is viral reactivation of Epstein Barr virus, due to different forms of immunosuppression including drug treatments or disease states. The risk of reactivation of certain viruses, including EBV, has been shown in other inflammatory conditions and in the transplant setting, however, few studies have determined the effect on viral titers of EBV after treatment with anti-TNF [13, 14]. Thus, we wanted to establish a case control study of Epstein- 
Barr virus (EBV) polymerase chain reaction (PCR) in patients treated with anti-TNFs and matched controls and a prospective study of potential PCR reactivation in patients starting anti-TNF treatment.

\section{MATERIALS AND METHODOLOGY}

\section{Study Population and Protocol}

The study population consisted of patients seen at St. Joseph's Hospital Rheumatology clinic, affiliated with the University of Western Ontario, which services a large referral area of approximately one million. The data are from patients who were seen serially by a rheumatologist and diagnosed with rheumatoid arthritis (meeting ACR criteria), ankylosing spondylitis (AS), psoriatic arthritis (PsA) or other inflammatory arthritis. AS was diagnosed according to the Modified New York Criteria (1984) where definite ankylosing spondylitis was present if the radiologic criterion was present in addition to at least one clinical criterion (which included low back pain, limitation in lumbar spine motion or limitation in chest expansion) [15]. Psoriatic arthritis was diagnosed clinically using the ClASsification of Psoriatic ARthritis (CASPAR) criteria (inflammatory joint disease with at least 3 points from the following features: current psoriasis; a history of psoriasis; a family history of psoriasis; dactylitis; juxta-articular new bone formation; rheumatoid factor negativity; and nail dystrophy) [16]. All diagnoses were made by a rheumatologist. Patients were recruited over a 6-month period and had at least two consecutive visits within an 18 month period. The study design consisted of two parts: one part was prospective and the second was a case control study. For the prospective study, patients with an inflammatory arthritis (RA, PsA, AS or other inflammatory arthritis) who were about to start anti-TNF treatment for their rheumatologic disease were recruited. Patients who were previously exposed to anti-TNF treatments or other biologics were excluded. For the case control study, similar diagnostic criteria were used to include patients, and patients were either taking a biologic (cases) or had never been exposed to a biologic treatment (controls). Patients were required to be treated with an anti-TNF biologic (etanercept, infliximab or adalimumab) for a minimum of two months. The cases were matched according to age, disease type, disease duration (within three years) and gender. The exclusion criteria were those patients not willing or unable to give consent. The type of treatment, including biologics, DMARDs and steroids, as well as baseline Health Assessment Questionnaire (HAQ) score, was recorded at the initial visit. Ethics approval was obtained locally through The University of Western Ontario Health Sciences Research Ethics Board.

After signing a consent form, IgG levels of EBV were tested in patients receiving biologics (cases) compared to patients who were biologic naïve (controls). All samples were run at the same time with the lab unaware of exposure status. Antibody (IgG class) to EBV viral capsid antigen was detected using enzymelinked immunosorbent assay technique. Viral load EBV DNA was amplified with specific primers using PCR on all IgG positive samples. No one had a history of a recent EBV infection; therefore, IgM was not tested.

\section{Analysis}

The frequency of EBV positivity (IgG) was measured and expressed as a percentage. EBV $\operatorname{IgG}>1.1$ was defined as a positive result; 1.1 was defined as an arbitrary cutoff measurement as positive according to the manufacturer (Trinity Biotech, Canada). EBV status was compared between cases and controls in the entire group as well as disease-based subsets. Chi square tests were used for the analysis between cases and controls. For the prospective study the proportion of patients who were sero-converting and with PCR activity between 6 and 18 months after antiTNF therapy was started was determined. Viral reactivation was defined as the presence of viral DNA in the plasma. Viral DNA was expressed as a quantitative variable using PCR as number of copies detected per reaction volume. $P$ values of $<0.05$ were considered statistically significant.

\section{RESULTS}

The mean age was 60 years (SD 13.2) and the mean disease duration was 11.7 years. The majority of patients were women $(83 \%)$. A total of 122 patients were recruited and had samples analysed for this study (72 taking anti-TNF and 50 controls). Ninety-nine patients had RA, 6 had AS, 12 had PsA, and there were 5 patients with other inflammatory arthritis. In terms of immunosuppressant therapy, the majority of patients (92\%) were using DMARD therapy, $50 \%$ non-steroidal anti-inflammatory drugs and $16 \%$ prednisone. The baseline HAQ score was $0.97+/-0.76$ (SD), with 0 corresponding to no disability and 3 to severe disability. None of the patients reported a history of malignant conditions nor did any of the patients have a history of EBV related infection.

For the viral studies, 93\% were seropositive for IgG EBV (98\% in the controls, and $90 \%$ in the cases) and there was no significant difference between cases and controls $(p=0.14)$. The results were similar according to each disease subtype; the majority of patients were seropositive for IgG EBV. Table 1 provides the percentage of patients with positive IgG EBV in cases and controls and according to the type of disease. Although some disease subsets are limited by smaller sample sizes, the data suggest that the majority of patients are seropositive for EBV. For the prospective study, quantitative PCR was only positive in one sample at 159 copies in a male with RA taking infliximab, but upon confirmatory testing of the same sample, it was actually negative. Fig. (1) shows the real time PCR amplification curve for EBV in a positive representative sample compared to a patient sample, which is negative. The results did not differ according to disease subtype (RA, PsA, AS or other inflammatory arthritis), as all of the viral PCR tests were negative. The rate of EBV in our centre (as known by transplant donors) is $>90 \%$. In the prospective study, all patients were EBV (IgG) positive; however, no one had positive viral PCR at the follow-up time points (6 months and 18 months).

\section{DISCUSSION}

It has been shown that various types of immunosuppression may induce viral reactivation, in particular EBV. Moreover, this reactivation of latent EBV may lead to the development of lymphoma. Our results show that the prevalence of EBV in this rheumatology practice (93\%) was similar to that seen in the transplant population at our centre. The positivity for EBV seen in this study is higher than expected, but unrelated to anti-TNF exposure. EBV infects nearly all humans by the 
Table 1. Percentage of IgG Positive EBV Patients Between Patients Treated with anti-TNF Agents (Cases) and Controls

\begin{tabular}{|c|c|c|c|c|c|}
\hline & \multicolumn{2}{|c|}{ Treated with Biologic (Anti-TNF) } & \multicolumn{2}{|c|}{ Biologic Naive } & \multirow{2}{*}{ p-Value } \\
\hline & $\mathbf{n}$ & IgG EBV & $\mathbf{n}$ & IgG EBV & \\
\hline Entire Study Population & 72 & $90 \%$ & 50 & $98 \%$ & 0.1 \\
\hline RA Only & 53 & $91 \%$ & 46 & $98 \%$ & 0.2 \\
\hline AS Only & 6 & $67 \%$ & 0 & - & - \\
\hline PsA Only & 9 & $100 \%$ & 3 & $100 \%$ & - \\
\hline $\begin{array}{c}\text { Other Inflammatory } \\
\text { Arthritis Only }\end{array}$ & 4 & $100 \%$ & 1 & $100 \%$ & - \\
\hline
\end{tabular}

$\mathrm{RA}=$ rheumatoid arthritis, $\mathrm{AS}=$ ankylosing spondylitis, PsA = Psoriatic arthritis.

\section{Amplification Curves}

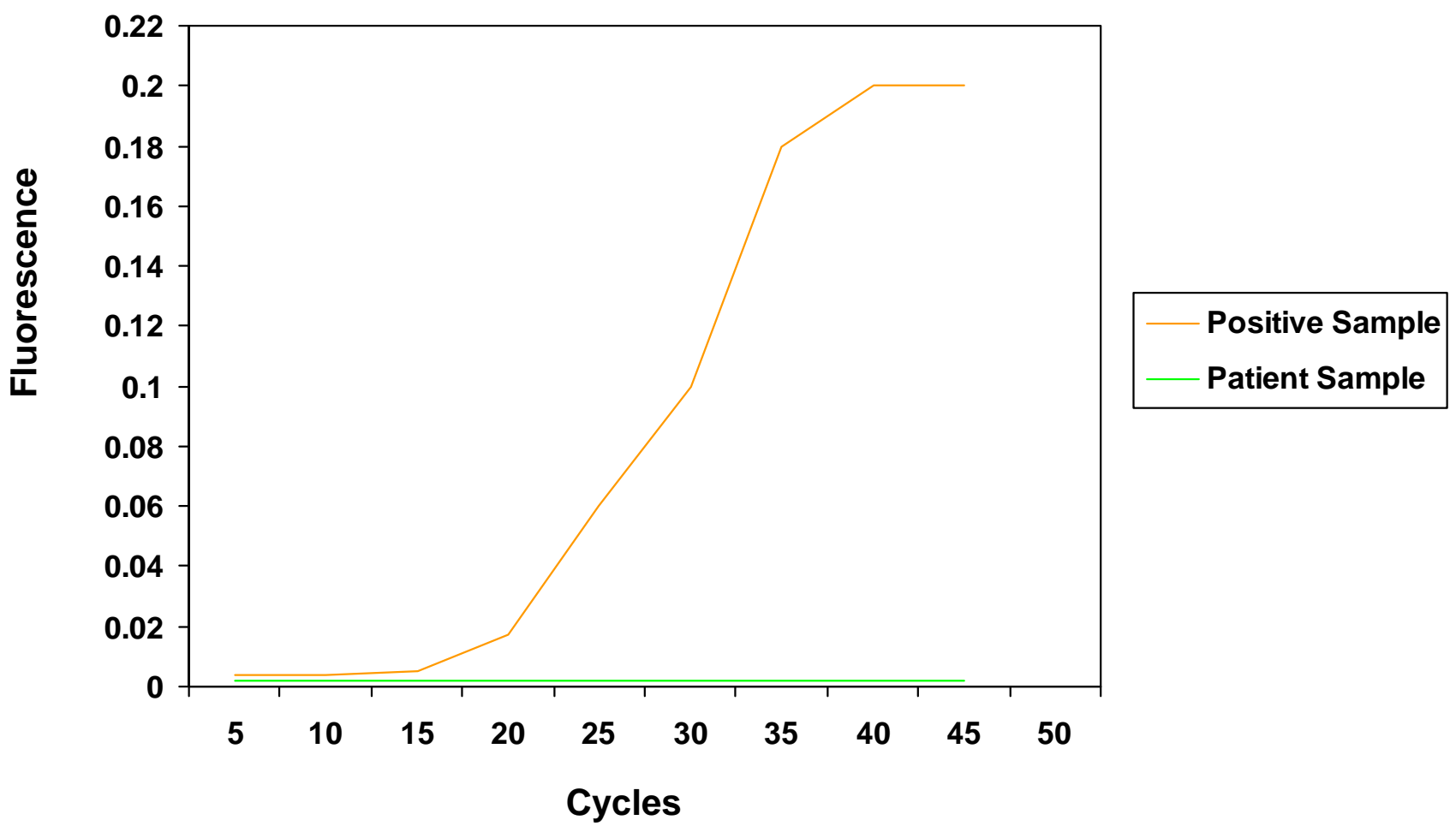

Fig. (1). Real time PCR analysis for quantitative EBV detection.

time they reach adulthood and the virus remains latent and resides in B lymphocytes. Healthy carriers can have approximately 1 to 50 infected cells per million leukocytes and latent infection is thought to be due to the limited expression of viral proteins to avoid immune recognition and detection [17]. However, in patients that are immunosuppressed a broader spectrum of viral proteins can be expressed allowing for further viral replication [17]. Patients with RA are considered immunosuppressed, thus this may explain the high rate of EBV positivity seen in the case control study $[11,12]$.

The evidence to support an increased risk of lymphoma in patients treated with anti-TNF agents is still under scrutiny and the mechanism for the contributory role of anti-
TNFs for oncogenicity is speculative $[8,14,18]$. The findings of this study are similar to the data from a study by TorreCisneros [19]. In a cohort of 15 patients with refractory forms of RA being treated with infliximab for 6 weeks, there was only one patient who developed viral reactivation of human herpesvirus (HHV)-6 after the onset of the anti-TNF. None of the patients developed reactivation of EBV, even though latent EBV virus was detected in $46.6 \%$ of patients. Our results suggest that anti-TNFs do not increase the risk of viral reactivation of $\mathrm{EBV}$, and that there may be other mechanisms of immunosuppression related to the development of lymphoma in RA patients.

The immunosuppressant drugs methotrexate and azathioprine have been investigated and may alter immune function and 
surveillance mechanisms and lead to the development of lymphoproliferative disorders in patients with RA. Discontinuation of methotrexate has been followed by a regression of lymphoma in numerous case reports, thus providing some evidence for a link, however there are conflicting results [14]. For example, Wolfe and Michaud prospectively studied 18,572 patients with RA and did not find an increased SIR for lymphoma in patients receiving methotrexate compared to those not exposed to methotrexate. In our study, the majority of patients were taking a DMARD that included methotrexate and this could confer additional immunosuppression leading to EBV positivity. There is some evidence that Crohn's patients treated with azathioprine and 6mercaptopurine may have an increased risk of developing lymphoma. Losco et al. report a case study of a patient with Crohn's disease treated with azathioprine and infliximab who developed diffuse large B cell lymphoma and EBV was detected (via PCR) in the neoplastic cells [13]. In contrast, the degree of immunosuppression to induce viral reactivation of EBV was not seen in this study, and there may be some amount of immune preservation in these patients that is not seen in other populations, such as organ transplantation.

In the transplant setting, for example, EBV associated post-transplant lymphoproliferative disorders (PTLD) are thought to be due to the transformation of the host lymphocytes, from the donor organ in EBV-seronegative patients, or a combination of donor and recipient EBV isolate in seropositive patients [14]. As a result, there is an expansion of multiple lineages of EBV transformed B cells, and the expression of a number of latent EBV oncogenes. This mechanism is supported by the high levels of EBV DNA in the cellular and cell-free fractions of blood in patients with PTLD [17]. Furthermore, EBV viral load in plasma acts as a marker of tumour burden in patients with sporadic EBV-related lymphomas including B cell, T cell, Natural killer cell and Hodgkin's subtypes [17]. In this mechanism of oncogenicity, the patient's immune system is unable to control latent EBV infection and impaired cellular immunity allows for the reactivation of EBV, leading to the development of lymphoma. Rohr et al. reported two cases of EBV associated lymphomas after solid organ transplant and found different EBV expression patterns associated with PTLD and infectious mononucleosis [20]. It has also been shown that EBV DNA levels increase before a patient becomes symptomatic due to PTLD, thus EBV viral load testing is done as a screening measure in patients at highest risk for PTLD [21]. Overall, the data from the transplant population suggests that immunosuppression is a critical risk factor for the development of EBV positive lymphomas. It is known that RA patients face some impairment in immunity that can lead to the development of serious bacterial infections and granulomatous infections, however, the necessary degree of immunosuppression to lead to an EBV associated lymphoma is unknown in this population $[6,10]$. In addition, the specific mechanism of immunosuppression has yet to be determined.

Limitations to this study should be reviewed and understood. First, our study included only several hundred patients, and therefore is underpowered to detect an excessive risk of a rare event, such as the development of lymphoma. The overall risk of lymphoma in patients with RA is conflicting in the literature. Wolfe and Michaud have attempted to address the possible risk of lymphoma in patients with RA using anti-TNF therapy. From the 19,591 patients in the National Data Bank for Rheumatic Diseases (NDB) registry, the authors found that there was no increase in the risk of lymphoma in patients treated with anti-TNF agents (odds ratio [OR] 1.0, 95\% CI 0.6-1.8 [P0.875]) [12]. These results are in contrast to a meta-analysis of randomized clinical trials (RCT) data conducted by Bongartz et al. using infliximab or adalimumab in RA. A total of 10 lymphomas in 3493 patients treated with anti-TNFs were identified compared to no lymphomas in 1,512 non-anti-TNF treated patients. The pooled odds ratio for malignancies in anti-TNF treated patients was $3.3(95 \%$ CI 1.2-9.1), indicating that there was a significantly higher risk of malignancy in patients with RA treated with anti-TNF therapy [8]. Bongartz et al. recently performed a second meta-analysis of RCTs of studies involving etanercept for a study duration of 12 weeks. Twenty-six patients in the etanercept group were diagnosed with a malignancy [incidence rate (IR) 10.47/1000 Patient Years (PY)] compared to 7 patients in the control group (IR 6.66/1000 PY), which was not significantly different between groups [22]. In a population based cohort study by Askling et al., the risk of lymphoma in patients with RA treated with antiTNFs was tripled (SIR $=2.9,95 \%$ CI 1.3 to 5.6 ) compared to the general population, however, when adjusted for sex, age, and disease duration, the risk of lymphoma after treatment with anti-TNF agents was no higher compared to the other RA cohorts [11]. Thus, the risk of lymphoma in patients using anti-TNF therapy for RA is still debatable making the search for potential mechanisms challenging in this population. We note also, that these previous studies have focused on the RA population whereas our study includes patients with other inflammatory conditions, AS and PsA. This heterogeneity within the patient population may have affected our results. Another limitation is that our observation period of 18 months may have been too short to observe viral reactivation of EBV. Given the unknown duration of development of EBV related lymphoproliferative diseases, it is premature to rule out the potential of EBV reactivation as a mechanism for the risk of developing lymphoma. As a result, we may have failed to identify some of the cases of EBV reactivation and this must be considered when interpreting our results.

\section{CONCLUSION}

In conclusion, it seems unlikely that EBV is reactivated in patients with RA taking anti-TNF agents, given the small sample size and relatively short timeframe from the initiation of biologic therapy in this study. To understand the potential risk of lymphoma in patients with rheumatoid arthritis, the degree of immunosuppression necessary to induce viral reactivation of EBV must include considerations of the underlying disease, other forms of immunosuppressant therapy and the exact duration for EBV infection. It appears that the patients in this study taking anti-TNFs were not completely immunocompetent, but also not as immunosuppressed as transplant patients to induce EBV reactivation. However, due to the serious implications of lymphoma and the lack of long term data with anti-TNF therapies, physicians still need to warn patients of the potential risk. At present, we would also recommend that 
screening tests for EBV are not necessary in patients with inflammatory disorders, such as rheumatoid arthritis, who are now candidates for anti-TNF therapies. Finally, future studies could help address the pathogenic mechanisms in other illnesses associated with anti-TNF therapies, such as serious infections and reactivation of tuberculosis to help elucidate potential mechanisms of immunosuppression.

\section{ACKNOWLEDGEMENT}

The authors would like to thank Gillian Ouimet for her assistance in the preparation of this manuscript.

\section{REFERENCES}

[1] van de Putte LB, Rau R, Breedveld FC, et al. Efficacy and safety of the fully human anti-tumour necrosis factor alpha monoclonal antibody adalimumab (D2E7) in DMARD refractory patients with rheumatoid arthritis: a 12 week, phase II study. Ann Rheum Dis 2003; 62: 1168-77.

[2] Lipsky PE, van der Heijde DM, St Clair EW, et al. Infliximab and methotrexate in the treatment of rheumatoid arthritis. Anti-Tumor necrosis factor trial in rheumatoid arthritis with concomitant therapy study group. N Engl J Med 2000; 343: 1594-602.

[3] Furst DE, Schiff MH, Fleischmann RM, et al. Adalimumab, a fully human anti tumor necrosis factor-alpha monoclonal antibody, and concomitant standard antirheumatic therapy for the treatment of rheumatoid arthritis: results of STAR (Safety Trial of Adalimumab in Rheumatoid Arthritis). J Rheumatol 2003; 30: 2563-71.

[4] Hochberg MC, Lebwohl MG, Plevy SE, Hobbs KF, Yocum DE. The benefit/risk profile of TNF-blocking agents: findings of a consensus panel. Semin Arthritis Rheum 2005; 34: 819-36.

[5] Primer on the rheumatic diseases. Atlanta: Arthritis Foundation, 2001.

[6] Kroesen S, Widmer AF, Tyndall A, Hasler P. Serious bacterial infections in patients with rheumatoid arthritis under anti-TNFalpha therapy. Rheumatology (Oxford) 2003; 42: 617-21.

[7] Wolfe F, Michaud K. Lymphoma in rheumatoid arthritis: the effect of methotrexate and anti-tumor necrosis factor therapy in 18,572 patients. Arthritis Rheum 2004; 50: 1740-51.

[8] Bongartz T, Sutton AJ, Sweeting MJ, Buchan I, Matteson EL, Montori V. Anti-TNF antibody therapy in rheumatoid arthritis and the risk of serious infections and malignancies: systematic review and meta-analysis of rare harmful effects in randomized controlled trials. JAMA 2006; 295: 2275-85.

[9] Leombruno JP, Einarson TR, Keystone EC. The safety of antiTumor Necrosis Factor treatments in rheumatoid arthritis: meta and exposure adjusted pooled analyses of serious adverse events. Ann Rheum Dis 2009; 68(7): 1136-45.

[10] Feldmann M, Elliott MJ, Woody JN, Maini RN. Anti-tumor necrosis factor-alpha therapy of rheumatoid arthritis. Adv Immunol 1997; 64: 283-350.

[11] Askling J, Fored CM, Baecklund E, et al. Haematopoietic malignancies in rheumatoid arthritis: lymphoma risk and characteristics after exposure to tumour necrosis factor antagonists. Ann Rheum Dis 2005; 64: 1414-20.

[12] Wolfe F, Michaud K. The effect of methotrexate and anti-tumor necrosis factor therapy on the risk of lymphoma in rheumatoid arthritis in 19,562 patients during 89,710 person-years of observation. Arthritis Rheum 2007; 56: 1433-9.

[13] Losco A, Gianelli U, Cassani B, Baldini L, Conte D, Basilisco G. Epstein-Barr virus-associated lymphoma in Crohn's disease. Inflamm Bowel Dis 2004; 10: 425-9.

[14] Tran H, Nourse J, Hall S, Green M, Griffiths L, Gandhi MK. Immunodeficiency-associated lymphomas. Blood Rev 2008; 22 . 261-81.

[15] Van der Linden S, Valkenburg HA, Cats A. Evaluation of diagnostic criteria for ankylosing spondylitis. A proposal for modification of the New York criteria. Arthritis Rheum 1984; 27: 361-68.

[16] Taylor W, Gladman D, Helliwell P, Marchesoni A, Mease P, Mielants H. CASPAR Study Group. Arthritis Rheum 2006; 54(8): 2665-73.

[17] Gulley ML, Tang W. Laboratory assays for Epstein-Barr virusrelated disease. J Mol Diagn 2008; 10: 279-92.

[18] Whelan P. Link between rheumatoid arthritis and cancer. Z Rheumatol 2006; 65: 497-2, 504.

[19] Torre-Cisneros J, Del Castillo M, Caston JJ, Castro MC, Perez V, Collantes E. Infliximab does not activate replication of lymphotropic herpesviruses in patients with refractory rheumatoid arthritis. Rheumatology (Oxford) 2005; 44: 1132-5.

[20] Rohr JC, Wagner HJ, Lauten M, et al. Differentiation of EBVinduced post-transplant Hodgkin lymphoma from Hodgkin-like post-transplant lymphoproliferative disease. Pediatr Transplant 2008; 12: 426-31.

[21] Reijasse D, Le Pendeven C, Cosnes J, et al. Epstein-Barr virus viral load in Crohn's disease: effect of immunosuppressive therapy. Inflamm Bowel Dis 2004; 10: 85-90.

[22] Bongartz T, Warren FC, Mines D, Matteson EL, Abrams KR, Sutton AJ. Etanercept therapy in rheumatoid arthritis and the risk of malignancies. A systematic review and individual patient data meta-analysis of randomized controlled trials. Ann Rheum Dis 2009; 68(7): 1177-83 